\title{
Cluster based Routing Protocol for Cognitive Radio Wireless Mesh Networks
}

\author{
Pavan Kumar $T^{1}$ Ramesh Babu B ${ }^{2}$ Rajasekhar Rao K ${ }^{1}$ Dinesh Gopalni ${ }^{2}$ \\ ${ }^{1}$ Department of Computer science and Engineering \\ K L University \\ ${ }^{2}$ Department of Computer Engineering \\ Malaviya National Institute of Technology Jaipur
}

\begin{abstract}
Cognitive wireless mesh networks (WMN's) are an emerged technology has made rapid changes in communication world. A wireless mesh network consists of mesh routers and mesh clients, in which mesh clients are highly mobile; due to this it should transmit continuous signaling messages to the gateway for their location registration. By transmitting signaling messages continuously more bandwidth is utilized so the overall performance of the network will be degraded. To overcome this problem we are introducing a new concept called clustering, which includes both static and dynamic clustering for mesh routers and mesh clients. By using these clustering algorithms we can reduce the signaling messages, so the overall network performance will be increased.
\end{abstract}

\section{Keywords}

Wireless mesh network (WMN), static clustering, dynamic clustering, mesh router (MR), mesh client (MC), cluster head $(\mathrm{CH})$, gateway $(\mathrm{GW})$.

\section{INTRODUCTION}

Wireless mesh networks (WMN) are highly used low cost networks. Due to their high connectivity and better performance people prefer these types of networks everywhere like broadband home networking, community and neighborhood networks, enterprise networks and building automation.[1] Mesh connectivity substantially improves network performance like load balancing, fault tolerance, protocol efficiency and throughput. The WMN support adhoc networks and have a capability of self-healing, self-forming and self-organization [1][20][21]. WMN consists of static wireless mesh routers which are also known as access points (AP's) [2], these static mesh routers will form the backbone of WMN and serve the mesh and conventional clients. Each AP connects mobile nodes to the wired network through multihop wireless routing. The mesh nodes are directly connected to the wired network through AP [4]. There are 3 different types of architectures in wireless mesh networks:

\section{1.) Infrastructure/ backbone WMN's \\ 2.) Client WMN's \\ 3.) Hybrid WMN's}

Infrastructure/backbone WMN's are most commonly used in community and neighborhood networks which are able to build infrastructure meshing. [1] [21] In this type of meshing the routers are placed on the top of the buildings which provide the internet access to the person inside the building and people on the roads. The client wireless meshing is mainly used to provide peer to peer networks to the clients. In this type of meshing the client nodes establish the actual network to perform routing and Configurations in order to provide user applications to the customers. Compared with the infrastructure/backbone WMN's the end user requirements are increased to perform additional functionalities like selfhealing and self configuring. The combination of infrastructure/ backbone WMN's and client WMN's resulted as hybrid WMN's. This hybrid WMN's is mainly used to provide access to the cellular networks, WI-Fi, WiMax, sensor networks. Compared to the infrastructure and client meshing the routing capabilities increase in hybrid WMN's which provide the better connectivity and coverage. [3][4][5]

\subsection{Cognitive Radio Networks:}

Next Generation (XG) communication networks, also known as Dynamic Spectrum Access Networks (DSANs) as well as cognitive radio networks will provide high bandwidth to mobile users through heterogeneous wireless architectures and dynamic spectrum access techniques. Before discussing about the cognitive radio networks let us know about cognitive radio. Cognitive radio means a radio system whose parameters is changing dynamically according to the external environment. By using the several cognitive radios' in the network they built cognitive radio networks. Cognitive radio networks is the developing area of wireless technology the main aim of the cognitive radio networks is increasing of the spectrum utilization [6][7].

The network mainly consisting of two types of users they are licensed users and unlicensed users. Licensed users are also known as primary users (PU) and unlicensed users are known as secondary user (SU). SU's access spectrum conditionally that means when primary users are inactive [8].

Cognitive radio had two characteristics they are Cognitive capability, Reconfigurability. Cognitive capability means the ability of the radio that can sense the environment. Cognitive capability gives the awareness of the spectrum whereas reconfigurability configuration of the radio to that environment (dynamically programmed).

The cognitive capability of a cognitive radio allows real time communication with its environment to find considerable communication parameters and adapt to the dynamic radio environment.

The steps are in the cognitive cycle mainly consisting of spectrum sensing, spectrum analysis and finally spectrum decision. 


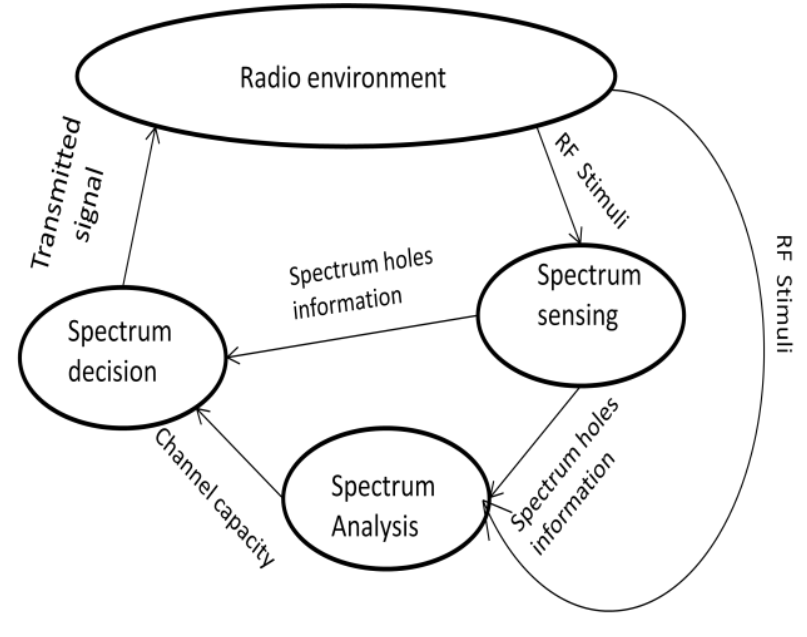

Figure-1: Cognitive wireless networks cycle

1. Spectrum sensing: A cognitive radio observes the available spectrum bands, captures their data, and then finds the white spaces.

2. Spectrum analysis: The characteristics of the spectrum holes that are detected through spectrum sensing are estimated.

3. Spectrum decision: A cognitive radio finds the data rate, the transmission mode, and the Bandwidth of the transmission. Then, the appropriate spectrum band is selected according to the Spectrum properties and user requirements.

Reconfigurability is the capability of arranging parameters for the transmission on the fly without any changes on the hardware components. This Capability of cognitive WMN is able to conform easily to the dynamic radio environment. There are several reconfigurable parameters as follows:

- Operating frequency: A cognitive radio has the ability of changing the operating frequency.

- Modulation: A cognitive radio should reconfigure the modulation scheme adaptive to the user requirements and channel conditions.

- Transmitting power: Transmission power can be reconfigured within the power constraints.

- Communication Technology: A cognitive radio can also be used to provide interoperability among different communication systems.

Wireless mesh networks with the cognitive capability to improve the functioning of the network.

\section{RELATED WORK}

In [1] [2], AODV protocol is used for cognitive wireless mesh networks by calculating the route selection and channel assignment. In case of small and static networks AODV protocol is more useful and we can use the bandwidth efficiently. WMN's consist of mesh routers and mesh clients, in mesh routers we can use the AODV protocol but as the mesh nodes are highly mobile so we can't use AODV protocol directly into an electronic network. So we are introducing clustering algorithms to divide the network into a group of clusters and then we adopt AODV in a network [17].The proposed clustering algorithms are static clustering algorithm for static mesh routers and dynamic clustering algorithm used for mesh clients.

\subsection{Signaling problem in WMN between mesh clients:}

The main issues in WMN is, if the nodes are more in a network all the nodes will access the GW at a time, so every node will send us signaling messages continuously as the GW receives a number of requests it cannot serve to all nodes at a time. As the node keeps on sending signaling messages the energy consumption will be more and to reduce this type of signaling problem we are dividing the whole network into clusters. By dividing the network into clusters each cluster will have a cluster head which is elected by the electoral process. So this $\mathrm{CH}$ will manage the respected cluster and access the GW to serve his clients. The clustering will be done for both static mesh routers and mesh clients. The static clustering will be done in between static mesh routers which are able to form the backbone of the network to serve the mesh clients. Dynamic clustering is done in between mobile nodes. This type of clustering is difficult compared to static clustering because mesh clients are highly mobile and as the node changes its position it has to register to another AP, for this the nodes have to calculate the delay between the nearest mesh router and the gateway through the $\mathrm{CH}$, as this delay is more then it will register to nearest mesh router otherwise it registers to the GW. By adopting this type of clustering in between the static routes and dynamic nodes the overall performance of the network will be increased, and all the nodes in a network will be able to utilize the bandwidth efficiently.

\subsection{Protocol Overview:}

\subsubsection{Proactive (Table-Driven) Routing Protocols}

In case of proactive routing protocols every node in the network maintains one or more routing tables. The routing tables are updated regularly. When the topology changes in the network every node sends a broadcast message to the entire network, by this broadcast message the throughput of the network may be affected. The protocols such as DSDV (Destination Sequence Distance Vector), FSR (Fisheye State Routing) and OLSR (Optimized Link State Routing) are the examples of proactive routing protocols [13].

\subsubsection{Reactive (On-Demand) Routing Protocols}

In case of reactive routing protocols routes are established on demand that means when a node wants to send data to another node then only the routes are discovered, for discovering the routes floods the control messages in the network by using the global broadcast. When the route is established between the source and destination the data is transmitted. The advantage of reactive routing protocols is that this protocol requires less routing information and the disadvantage is that it produces huge control packets for route discovery when topology changes. The examples of this type of protocol are Dynamic Source Routing (DSR), Ad-hoc On Demand Routing (AODV) and Associatively Based Routing

\section{CLUSTER-BASED ROUTING PROTOCOL}

\subsection{Protocol Overview}

Please use a 9-point Times Roman font, or other Roman font with serifs, as close as possible in appearance to Times Roman in which these guidelines have been set. The goal is to have a 9-point text, as you see here. Please use sans-serif or non-proportional fonts only for special purposes, such as 
distinguishing source code text. If Times Roman is not available, try the font named Computer Modern Roman. On a Macintosh, use the font named Times. Right margins should be justified, not ragged.

\subsection{Static Clustering}

WMN's consists of mesh routers and mesh clients; the proposed static clustering algorithm is applicable for static mesh routers and clients.

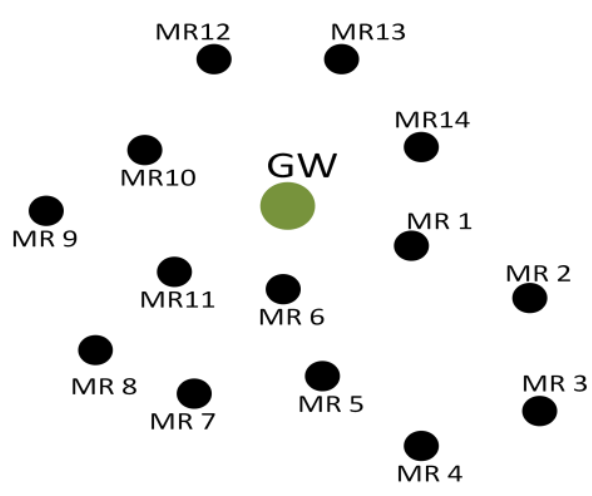

Figure 3: A sample WMN consists of static Wireless mesh routers

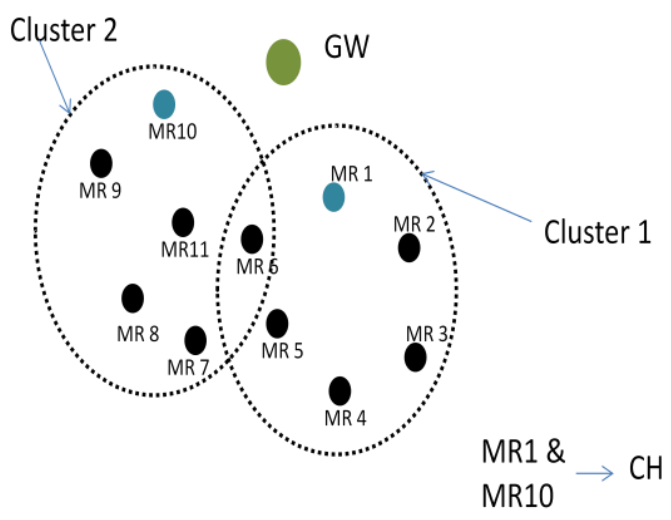

Figure 4: WMN after forming into clusters.

In the above figure the whole network is divided into 2 clusters using static clustering algorithm. The main idea behind static clustering algorithm is to increase the utilization of bandwidth in a network. In between these routers election process will be done for the election of cluster head $(\mathrm{CH})$. In the above network MR1 and MR10 are elected as cluster heads.

$\mathrm{U}_{\mathrm{n}}=$ Undecided node, $\mathrm{C}_{\mathrm{h}}=$ Cluster head, $\mathrm{C}_{\mathrm{m}}=$ Cluster member, Timeperoid $=\mathrm{t}$

$\mathrm{TH}_{\mathrm{m}}=$ Triggered HELLO message, $\mathrm{j}=$ number of hops, $\mathrm{L}=$ Link

$1=$ uni- directional

2=bi-directional

Step 1: when the node comes up

Step 2: if node is $U_{n}$ state

Step 3: Then $U_{n}$ broadcasts HELLO message $j$ hops away nodes ( $\mathrm{j}$ being set to 1 by default) Timer $1=t++$ seconds (where $\mathrm{t}=0,1,2,3,4, \ldots$ ..,n )

Step 4: when $C_{h}$ gets the HELLO message

Then $\mathrm{C}_{\mathrm{h}}$ replies with $\mathrm{TH}_{\mathrm{m}}$ $\begin{gathered}\text { Timer } \\ 1,2,3,4, \ldots \ldots \ldots \ldots, \mathrm{n})\end{gathered} \quad+\mathrm{t} \quad++\quad$ seconds where $\quad(\mathrm{t}=0$,

End if

Step 5: if (Timer 1=Timer 2)

Then $\quad \mathrm{U}_{\mathrm{n}} \rightarrow \mathrm{C}_{\mathrm{m}}$

Step 6: else if(Timer $1 \neq$ Timer 2 )

Then $\mathrm{U}_{\mathrm{n}}$ checks $\mathrm{L}=2$

$\mathrm{U}_{\mathrm{n}} \rightarrow \mathrm{C}_{\mathrm{h}}$

End if

End if

Step 7: if $L \neq 2$

repeat the procedure from Step 1

end if

When the node enters into the network it is in undecided state $\left(U_{n}\right)$, it broadcasts hello messages ' $j$ ' hops away from the node. Initially $\mathrm{j}$ value set to 1 , when the node broadcasts hello messages timer 1 begins. Where timer starts from $0,1,2 \ldots . n$. if the hello messages are received by cluster head $(\mathrm{CH})$ then it sends an triggered hello message to undecided node. Then it starts timer 2, If timer 1 is equal to timer 2 then it is elected as a cluster member, otherwise it checks the number of links to the other neighboring nodes if the links are maximum, it itself considers as a $\mathrm{CH}$. If the above condition fails i.e.., if the links are minimum then the process repeats from the beginning.

\subsection{Dynamic Clustering}

Please The network consists of mesh clients, as the mesh client moves randomly in the network they have to register the gateway every time when their location changes. So we are adopting a dynamic clustering algorithm between the mesh clients [3][19].

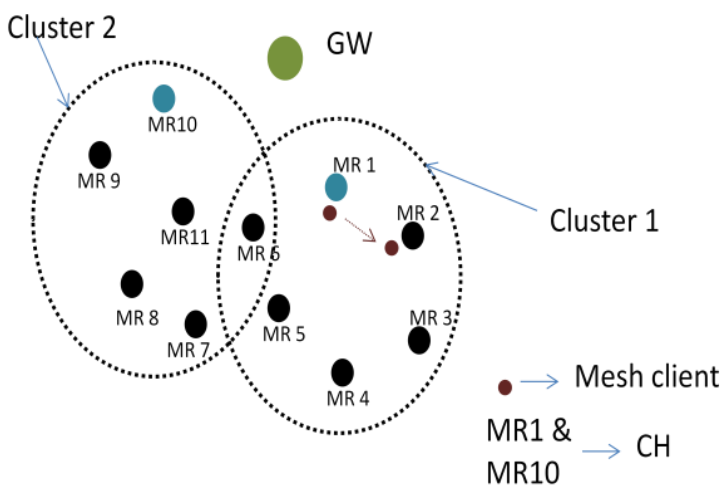

Figure 5: mesh client moving randomly from MR1 to MR2.

In the above figure mesh client moves from MR1 to MR2 where MR1 is the cluster head of the cluster 1 . Now the mesh client calculates the indirect distance between GW and new MR through $\mathrm{CH}$. If the maximum delay is greater than or equal to distance between new MR and GW, then it registers to the MR1, when this condition is satisfied then it calculates signaling cost (in terms of occupied wireless links by the signaling messages). If this condition fails then it directly registers to $\mathrm{GW}$. 


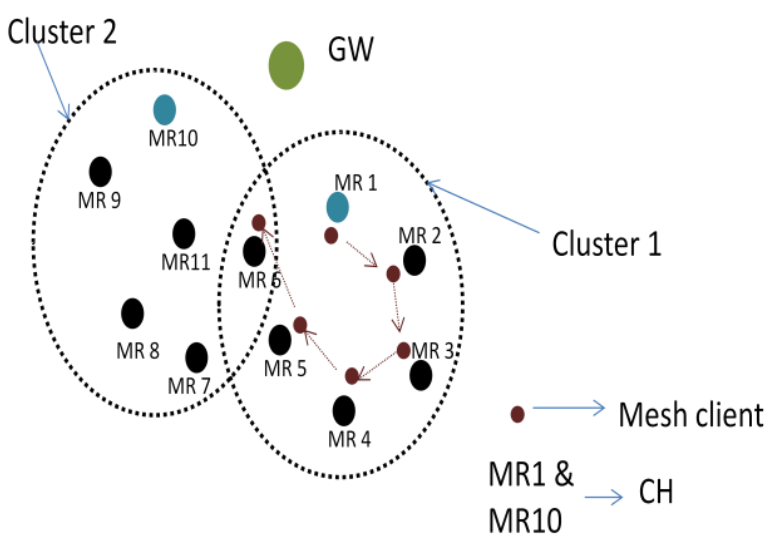

Figure 6: mesh client moving randomly from router to router.

In the above figure the mesh client is changing its position randomly so it will calculate the distance every time and it registers to the respective router.

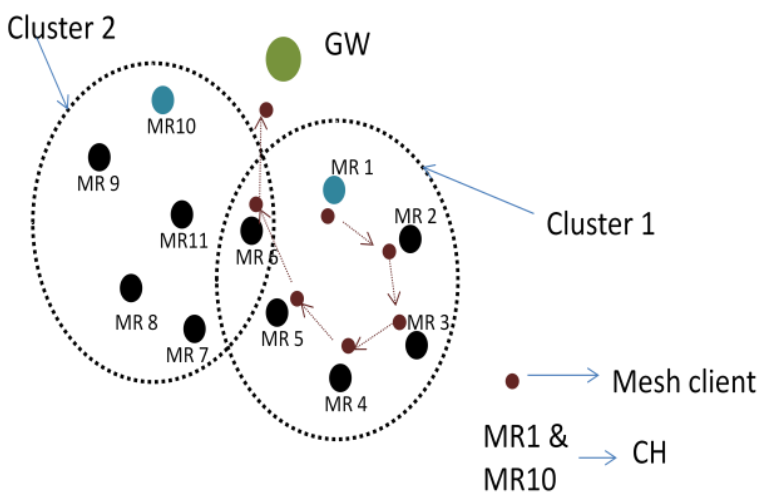

Figure 7: the mesh client moves out of the cluster.

In the above figure the mesh client moves out of the cluster then it calculates the indirect distance between GW and MR10 and the distance is less to the GW compared to MR10, so it registers directly to the $\mathrm{GW}$ as a individual client.

$\mathrm{M}_{\mathrm{C}}=$ Mesh Client, $\mathrm{M}_{\mathrm{R}}=$ Mesh Router, $\mathrm{C}_{\mathrm{H}}=$ Cluster Head, $\mathrm{GW}=$ Gateway, $\mathrm{D}_{\max }=$ maximum delay (in terms of hops), $\mathrm{d}(\mathrm{x}$, $\mathrm{y})=$ distance from $\mathrm{x}$ to $\mathrm{y}$,

$\rightarrow$ Enters into

Step 1: If $\left(\mathrm{M}_{\mathrm{C}} \rightarrow\right.$ new cluster $)$

New $M_{R}$ checks the existence of the current $C_{H}$ IP address in its routing table.

Step 2: If (address exists)

New $M_{R}$ calculates the shortest distance to the $C_{H}$ and to GW.

Step 3: If $\left(d\left(\right.\right.$ new $\left.\left.M_{R}, G W\right)>d\left(n e w M_{R}, C_{H}\right)\right) \& \&(d$ (new $\left.\left.\mathrm{M}_{\mathrm{R}}, \mathrm{C}_{\mathrm{H}}\right)+\mathrm{d}\left(\mathrm{C}_{\mathrm{H}}, \mathrm{GW}\right) \leq \mathrm{D}_{\text {max }}\right)$

Step 4: if $\left(\mathrm{n}\left(\mathrm{M}_{\mathrm{c}}\right.\right.$ for $\left.\mathrm{C}_{\mathrm{H}}\right) \leq \mathrm{MAX}$

Do a $\mathrm{C}_{\mathrm{H}}$ registration: new $\mathrm{M}_{\mathrm{R}}$ added to the cluster.

Else

$C_{H}$ elects the temporary $C_{H}$ where $n\left(\right.$ Links $\left.M_{C}=M A X\right)$

End if
Step 5: Do a GW registration: new cluster formation.

Step 6: $C_{H}$ renewal: new $M_{R}$ becomes the new $C_{H}$.

Step 7: Endif

Step 8: Endif

Step 9: Endif

\section{CONCLUSIONS}

In this paper, the proposed algorithms are called static clustering and dynamic clustering algorithms. The proposed algorithms are adopted at the mesh router level and client level, because the mesh clients are highly mobile nodes, so they have to use dynamic clustering at this level. In this paper, all mobile clients are at dynamic cluster. All mesh routers are at static cluster level, because of its mobility and transmission capabilities. By adopting these algorithms the overall performance of the network will be increased and energy consumption will be mitigated at the nodes. So the main issue of this paper i.e.., signaling messages are transmitted more by the clients to the GW are eventually reduced by applying these algorithms in a WMN.

\section{REFERENCES}

[1] Ian F. Akyildiz, Xudong Wang, Weilin Wang "Wireless mesh networks: a survey" Broadband and Wireless Networking (BWN) Lab, School of Electrical and Computer Engineering, Georgia Institute of Technology, Atlanta, GA 30332, USA January 2005

[2] "Hoteit, S.; Secci, S.; Langar, R.; Pujolle, G. "Strategic subchannel resource allocation for cooperative OFDMA Wireless Mesh Networks", Communications (ICC), 2012 IEEE International Conference on, On page(s): 440 $-444$

[3] Hatoum, A.; Aitsaadi, N.; Langar, R.; Boutaba, R.; Pujolle, G. "FCRA: Femtocell Cluster-Based Resource Allocation Scheme for OFDMA Networks", Communications (ICC), 2011 IEEE International Conference on, On page(s): 1 - 6.

[4] D. De Couto, D. Aguayo, J. Bicket, and R.Morris. "High-throughput path metric for multi-hop wireless routing."In MOBICOM, 2003

[5] Ian F. Akyildiz, Won-Yeol Lee, Mehmet C. Vuran, and Shantidev Mohanty. 2006. NeXt generation/dynamic spectrum access/cognitive radio wireless networks: a survey. Comput. Netw. 50, 13 (September 2006), 2127 2159. DOI $=10.1016 /$ j.comnet.2006.05.001 http://dx.doi.org/10.1016/j.comnet.2006.05.001

[6] Geng Cheng, Wei Liu, Yunzhao Li, and Wenqing Cheng, "Joint On-demand Routing and Spectrum Assignment in Cognitive Radio Networks" IEEE.ICC.2007.

[7] Xin C, Xie B, and Shen C. "A novel layered graph model for topology formation and routing in dynamic spectrum access networks"[C]. DySPAN 2005, Baltimore, MD, United States, 2005: 308-317

[8] Federal Communications Commission, "Spectrum Policy Task Force", Rep. ET Docket no. 02-135, Nov. 2002

[9] C. Perkins, E. Belding-Royer and S. Das "Ad hoc OnDemand Distance Vector (AODV) Routing.” RFC 3561, IETF Network Working Group, July 2003.

Else 
[10] T. Clausen and P. Jacquet "Optimized Link State Routing Protocol (OLSR).” RFC 3626, IETF Network Working Group, October 2003.

[11] D. S. J. de Couto, "High-throughput routing for multihop wireless networks", Ph.D. dissertation, MIT, 2004.

[12] D. Johnson, et al., "DSR: The Dynamic Source Routing Protocol for Multi-Hop Wireless Ad Hoc Networks," in In ad Hoc Networking, edited by Charles E. Perkins, Chapter 5, 2001, pp. 139-172.

[13] ] R. Draves, J. Padhye, and B. Zill, "Routing in multiradio, multi-hop wireless mesh networks", in ACM International Conference on Mobile Computing and Networking (MobiCom), Sept. 2004, pp. 114-128.

[14] Yang, Y. and Wang, J., "Design guidelines for routing metrics in multihop wireless networks", IEEE INFOCOM, 2008.

[15] C. E. Perkins and P. Bhagwat, "Highly dynamic Destination-Sequenced DistanceVector routing (DSDV) for mobile computers," SIGCOMM Comput. Commun. Rev., vol. 24, pp. 234-244, 1994.

[16] M. Gerla and J. T. chieh Tsai, "Multicluster, Mobile, Multimedia Radio Network," Journal of Wireless Networks, vol. 1, pp. 255-265, 1995.
[17] N. Mitton, A. Busson, and E. Fleury, "Self-Organization in Large Scale Ad Hoc Networks," in Proc. The Third Annual Mediterranean Ad Hoc Networking Workshop (Med-Hoc-Net 2004), Juni 2004, pp. 14-23.

[18] Y. Xu and W. Wang, "Topology Stability Analysis and Its Application in Hierarchical Mobile Ad Hoc Networks," IEEE Transactions on Vehicular Technology, vol. 58, no. 3, pp. 1546-1560, March 2009.

[19] "Hompage of Optimized Link State Routing Daemon (OLSRD)," 2010. [Online]. Available: http://www.olsr.org/

[20] UMIC-Mesh, "Hompage of the UMIC Wireless Mesh Testbed," 2010. [Online]. Available: http://www.umicmesh.net/.

[21] R. Battula, S. Vemuru, R. Kurra, P. Tummal, and J. Deka, "On-demand table-driven topo-aware routing protocol for wireless mesh networks," in Recent Trends in Networks and Communications (N. Meghanathan, S. Boumerdassi, N. Chaki, and D. Nagamalai, eds.), vol. 90 of Communications in Computer and Information Science, pp. 296-305, Springer Berlin Heidelberg, 2010. 